\title{
The Case against Power Utility and a Suggested Alternative: \\ Resurrecting Exponential Utility
}

\author{
Sami Alpanda \\ Geoffrey Woglom \\ Amherst College \\ Amherst College
}

July 2007

\begin{abstract}
Utility modeled as a power function is commonly used in the literature despite the fact that it is unbounded and generates asset pricing puzzles. The unboundedness property leads to St. Petersburg paradox issues and indifference to compound gambles, but these problems have largely been ignored. The asset pricing puzzles have been solved by introducing habit formation to the usual power utility. Given these issues, we believe it is time re-examine exponential utility. Exponential utility was abandoned largely because it implies increasing relative risk aversion in a cross-section of individuals and nonstationarity of the aggregate consumption to wealth ratio, contradicting macroeconomic data. We propose an alternative preference specification with exponential utility and relative habit formation. We show that this utility function is bounded, consistent with asset pricing facts, generates near-constant relative risk aversion in a cross-section of individuals and a stationary ratio of aggregate consumption to wealth.
\end{abstract}

Keywords: unbounded utility, asset pricing puzzles, habit formation JEL Classification: D810, G110 


\section{Introduction}

Exponential utility functions have been mostly abandoned from economic theory despite their analytical convenience. On the other hand, power utility, which is also highly tractable, has become the workhorse of modern macroeconomics and asset pricing. This apparent preference of power utility over exponential utility by the literature was mainly motivated by two important economic observations:

First, most empirical studies suggest risk aversion among individuals with different levels of wealth is roughly constant [Friend and Blume (1975)]. The exponential utility function given by

$$
U(C)=\frac{e^{-A C}}{-A}
$$

exhibits constant absolute risk aversion (equal to $A>0$ ), hence generates increasing relative risk aversion (IRRA). The cross-sectional implication of this is that wealthier people are more risk-averse and in their portfolio choice invest a smaller proportion of their wealth in the risk-free asset [Merton (1992)]. This prediction is counterintuitive and is contradicted by empirical studies. Constant relative risk aversion (CRRA), power utility which is given by

$$
U(C)=\frac{C^{1-\gamma}}{1-\gamma}
$$

on the other hand, exhibits constant relative risk aversion (equal to $\gamma>0$ ) and hence is immune from this criticism. ${ }^{1}$

The second observation motivating the use of CRRA power utility over exponential utility is that aggregate consumption to wealth ratio is stationary in the data.

\footnotetext{
${ }^{1}$ Merton for example states that he finds CARA utility "behaviorally less plausible than" CRRA due to their different portfolio choice implications (Merton [1969]).
} 
With exponential utility, optimal consumption is linear in wealth, but not proportional to wealth, hence their ratio is not stationary [Merton (1992)]. ${ }^{2}$ With CRRA power utility, optimal consumption is proportional to wealth and therefore consumption-wealth ratio is stationary and thus consistent with a balanced growth path.

Despite the aforementioned strengths, the CRRA, power utility also has its weaknesses. The best known weakness of the CRRA power utility is that it has undesirable asset pricing implications. In particular, it has a hard time matching the high risk-premium observed in the data unless one assumes very high values of relative risk aversion; this is known as the equity premium puzzle [Mehra and Precott (1985)]. In a Consumption-based Capital Asset Pricing Model (C-CAPM) with CRRA power utility, an asset is risky if the covariance of its returns with consumption growth is high. Agents prefer to hold assets which give high returns when times are bad (i.e. when consumption is low and marginal utility of consumption is high) and would like to avoid assets which give low returns when times are bad. To hold assets which co-vary positively with consumption growth, agents require a higher risk-premium.

Even if one accepts high relative risk aversion, the CRRA, power utility still generates problems, this time in accounting for the low risk-free rates observed in the data. With power utility, the risk aversion parameter $\gamma$ is also the inverse of the intertemporal elasticity of substitution. High risk aversion therefore implies a very small elasticity of intertemporal substitution: people who wish to avoid volatile consumption will also wish to avoid secular growth in consumption. Since consumption actually grows over time, people should be demanding high interest rates in compensation. The low risk-

\footnotetext{
${ }^{2}$ Note however that optimal consumption is linear in wealth, hence the consumption-wealth ratio does approach a constant asymptotically.
} 
free rates observed in the data are thus a puzzle; i.e. the risk-free rate puzzle [Weil (1989)].

The desirable properties of power utility have been preserved in spite of the puzzles by modifying the model to include habit formation. (see Sunderasan [1989], Constantinides [1990] and more recently, Campbell and Cochrane [1999]). These models employ a decreasing relative risk aversion (DRRA) form of power utility:

$$
U(C)=\frac{(C-Z)^{1-\gamma}}{1-\gamma} ; Z>0
$$

Where $Z$ is the so-called "habit" level of consumption and $C-Z$ is sometimes referred to as "surplus" consumption. The habit level can either be internally generated by individual past consumption, as in the models of Sunderasan (1989) and Constantinides (1990), or can be externally generated by aggregate consumption, as in Campbell and Cochrane (1999). This formulation can solve the asset pricing puzzles because secular growth in consumption leads to roughly proportional growth in surplus consumption, where $\gamma$ determines aversion to secular growth. Volatility in $C$ does not affect $Z$ and aversion to volatility is measured by $\gamma$ times the ratio of consumption to surplus consumption. ${ }^{3}$ With assumed surplus consumption between 10 and 20 percent, aversion to volatility can be 5-10 times the aversion to secular growth.,

\footnotetext{
${ }^{3}$ Relative risk aversion is $\gamma C /(C-Z)$

${ }^{4}$ Abel (1990) and Gali (1990) adopt a relative form of habit, where the argument of the utility function is $C / Z$, but they stick with power utility. This form of habit leads to a different solution to the risk-free puzzle, which we will detail below.

${ }_{5}^{5}$ Another idea was to separate the parameters governing risk aversion and the elasticity of intertemporal substitution directly by making utility recursive and non-separable across states of nature (Epstein and Zin [1991]).
} 
But power utility whether of the CRRA or DRRA flavors also has a long known weakness due to unboundedness. ${ }^{6}$ Unbounded utility can result in so-called St. Petersburg-style paradoxes where events with vanishingly small probabilities of occurrence have a finite effect on behavior. For example, for the familiar case of utility being unbounded from above, where agents choose to take on gambles with a large probability of considerable losses, but a tiny probability of infinite gains. Similarly, if utility is not bounded from below, vanishingly small probabilities of infinite losses may preclude agents from taking on an otherwise reasonable gamble [Arrow (1971)].

Exponential utility on the other hand is bounded and is consistent with the observed equity-premium without resorting to an extremely high risk aversion parameter. The weaknesses of power utility are the strengths of the exponential utility, just as the strengths of power utility are their weaknesses. But given the weaknesses of power utility, we believe it is worth trying to solve the weaknesses of exponential. In this paper we suggest an exponential utility with habit formation where utility is defined over the ratio of consumption to the habit level of consumption (relative habit):

$$
U(C, Z)=\frac{e^{-A C / Z}}{-A}
$$

This utility function preserves the strengths of the standard exponential utility function, but solves the weaknesses, namely ensures the stationarity of consumption relative to wealth and the near-constancy of relative risk aversion across the population with

\footnotetext{
${ }^{6}$ With $Z$ less than zero, power utility implies IRRA. While this utility function is bounded (for $\gamma>1$ ), it cannot solve the risk-free rate puzzle. One interpretation for this specification is that $-Z$ stands for housing services, as in Davis and Martin (2005). They conclude that housing cannot solve the asset pricing puzzles if consumption and housing services are not complementary. We will not consider the IRRA version of power utility further.
} 
different levels of wealth and solves the risk-free puzzle because of the absence of secular growth in $C / Z$.

Table 1 provides a brief synopsis on the utility functions we considered and summarizes the main points we made so far (an $\mathrm{X}$ in a particular box indicates that the corresponding utility function is in line with the corresponding issue):

\section{Table 1: Synopsis on Utility Functions}

\begin{tabular}{|l|c|c|c|c|c|c|}
\hline Utility & $\begin{array}{l}\text { Power with } \\
\text { no habit }\end{array}$ & $\begin{array}{l}\text { Power with } \\
\text { habit, C-Z }\end{array}$ & $\begin{array}{l}\text { Power with } \\
\text { relative } \\
\text { habit, C/Z }\end{array}$ & $\begin{array}{l}\text { Exp. with } \\
\text { no habit }\end{array}$ & $\begin{array}{l}\text { Exp. with } \\
\text { habit, } \\
\text { C-Z }\end{array}$ & $\begin{array}{l}\text { Exp. with } \\
\text { relative } \\
\text { habit, C/Z }\end{array}$ \\
\hline Bounded Utility & & $\mathbf{X}$ & $\mathbf{X}$ & $\mathbf{X}$ & $\mathbf{X}$ & $\mathbf{X}$ \\
\hline $\begin{array}{l}\text { Observed equity } \\
\text { premium }\end{array}$ & & $\mathbf{X}$ & $\mathbf{X}$ & & & $\mathbf{X}$ \\
\hline Risk-free Rate & $\mathbf{X}$ & $\mathbf{X}$ & & & $\mathbf{X}$ \\
\hline $\begin{array}{l}\text { Stationarity of } \\
\text { Cons. / Wealth }\end{array}$ & $\mathbf{X}$ & $\mathbf{X}$ & $\mathbf{X}$ & & & $\mathbf{X}$ \\
\hline $\begin{array}{l}\text { Similar RRA } \\
\text { across individuals }\end{array}$ & $\mathbf{X}$ & $\mathbf{X}$ & & & $\mathbf{X}$ \\
\hline
\end{tabular}

The next section re-examines a long-standing issue for unbounded power utility: what we call indifference to compound gambles. Samuelson (1963) appears to have disposed of this issue by convincing the profession that the special results of CRRA, power utility made sense. We show that more plausible results arise from IRRA utility functions, such as exponential utility. In section 3, we look at the equity premium puzzles in terms of the properties of the direct utility function of consumption and the indirect utility function with of wealth. This analysis allows us to explain how a simple,

\footnotetext{
${ }^{7}$ Sundaresan (1989) and Constantinides (1990).

${ }^{8}$ Abel (1990) and Gali (1990).

${ }^{9}$ Sundaresan (1989)
} 
exponential utility function solves our re-stated equity premium puzzle, and also shows that indirect utility function of the power utility habit formation model is inconsistent with the data. In section 4 , we introduce our version of the exponential utility with relative habit formation. We show the relationship between the direct and indirect utility function, and explain how relative habit eliminates the risk-free rate puzzle. In section 4 we conclude.

\section{Unboundedness and Indifference to Compound Gambles}

As already noted, CRRA preferences are subject to St. Petersburg-type paradoxes because of unbounded utility. A related problem of CRRA, power utility that is due to the unbounded utility, is what we call indifference to compound gambles. With preferences that exhibit constant relative risk aversion, an individual's choice for a gamble does not depend on the number of times the gamble is performed. Consider the gamble where wealth will increase by the factor $X>1$, with probability $P>1 / 2$ and will decrease by $1 / X$, with probability $(1-P)$ (local relative risk aversion is assumed to be greater than one). Assume that the values of $P$ and $X$ have been selected so that our subject is indifferent to the one-trial gamble

$$
E_{0}\left[V\left(W_{1}\right)\right]=P V\left(W_{0} X\right)+(1-P) V\left(W_{0} X^{-1}\right)=V\left(W_{0}\right),
$$

where $V\left(W_{0}\right)$ is the indirect utility function, the value of lifetime utility after one has optimized consumption and portfolio choice. The subscript on $W$ indicates the number of trials for the gamble. 
Now consider an alternative gamble where the outcomes are based on two binomial trials with the same values for $X$ and $P$. The subject's expected utility from this "compound" gamble is given by

$$
E_{0}\left[V\left(W_{2}\right)\right]=P^{2} V\left(W_{0} X^{2}\right)+2 P(1-P) V\left(W_{0}\right)+(1-P)^{2} V\left(W_{0} X^{-2}\right)
$$

We are not imagining the two gambles as being sequential, where the subject could choose whether to continue after observing the outcome of the first trial. Instead, we are asking our subject to commit ex ante to the outcomes of the two-trial gamble ${ }^{10}$

A constant relative risk aversion subject who is indifferent to the one-trial gamble will be also be indifferent to the outcomes based on two binomial trials because the utility consequences of the two-trial gamble are just the square of the one-trial gamble.

$$
V\left(W_{0} X^{a}\right)=V\left(W_{0}\right) V(X)^{a}, \text { for any } a
$$

Of course, an exactly similar argument can be made for a gamble based on 3 or more binomial trials where the outcomes are adjusted accordingly.

But herein lies the paradox: with $P>0.5^{11}$, the probability of a loss gets smaller and smaller as the number of trials increases. As the number of trials increases without limit, the probability of a loss approaches zero, but our CRRA subject will value this gamble as equal to the first gamble. While the probability of losses is going to zero, the maximum size of those losses is increasing without limit and the utility consequences of those losses have finite consequences with constant relative risk aversion. This strikes us as implausible as people do seem to take chances such as those in many everyday situations (e.g. driving, flying on an airplane). Arrow (1971) formalized this in the

\footnotetext{
${ }^{10}$ While Samuelson (1963) does not consider proportional gambles, he does assume a one trial gamble based on a sequence of trials. See his footnote 2 section 4 .

${ }^{11} P>0.5$ requires relative risk aversion greater than 1.
} 
principle that events with vanishingly small probabilities of occurrence should not have finite effects on behavior, and this principle is violated by CRRA power utility.

These implausible results are specific to constant relative risk aversion preferences. Preferences with increasing relative risk aversion can avoid these problems because they avoid the problem of unbounded utility at either high or low levels of wealth. For example, exponential utility and IRRA power utility functions are bounded and exhibit increasing relative risk aversion: For subjects with these preferences a twotrial gamble is preferred to no gamble, even when the subject is indifferent between the one-trial gamble and no gamble. With increasing relative risk aversion (as is shown in the Appendix):

$$
\begin{aligned}
& V\left(W_{0} X^{a}\right)>V\left(W_{0}\right) V(X)^{a}, \text { so that, } \\
& E_{0}\left[V\left(W_{2}\right)\right]>E_{0}\left[V\left(W_{1}\right)\right]=V\left(W_{0}\right)
\end{aligned}
$$

More generally, our increasing relative risk aversion subject will accept any gamble, where $P>0.5, X>1$ if one allows a sufficient number of trials. ${ }^{12}$ DRRA power utility, however, makes the situation worse because: 1) A DRRA subject would reject a two-trial gamble, if she were indifferent to the one trial gamble; 2) A gamble with any probability of hitting the capitalized value of habit consumption will be rejected, no matter how favorable the odds because the utility consequence of hitting the capitalized value is unbounded.

\footnotetext{
${ }^{12}$ Samuelson's (1963) in an article entitled the "Risk and Uncertainty: A Fallacy of Large Numbers" has a sharp criticism of a faculty lunchroom colleague who believes the number of times the gamble is performed matters by the "Law of Large Numbers." Samuelson is careful (footnote 1 section 8), however, to note: "[My theorem] does not state one must always refuse a sequence if one refuses a single venture: if at higher income levels the single tosses become acceptable, and at lower levels the penalty of losses does not become infinite, there might well be a long sequence that is optional."
} 
As noted in the introduction, there are problems with exponential utility, but this section has shown that there are also problems with the CRRA and DRRA versions of power utility. As a result, we will explore the advantages of exponential utility and ways of avoiding its disadvantages

\section{The Equity Premium Puzzle:}

Since the development of consumption-based portfolio choice models and Mehra and Prescott's (1985) path breaking work, most attention has focused on the asset-pricing implications of Euler equations based on the direct utility function. We believe it is important to look at this issue in terms of all of the implied properties of the model, including implications for the indirect utility function and the consumption function.

Consider the following portfolio allocation problem:

$$
\begin{aligned}
V[W(t)]= & \max _{C(t), \phi(t)} E \int_{0}^{\infty} e^{-\rho t} U[C(t)] d t \\
& \text { s.t. } \frac{d W}{d t}=\{\phi(t)[\alpha(t)-r]+r\} W(t)-C(t) \\
& \text { s.t. } \log \alpha(t) \sim N\left(\mu, \sigma^{2}\right)
\end{aligned}
$$

where $\rho$ is the pure rate of time preference; $\phi$ is the share in the risky asset; $\alpha$ is the return on the risky asset, $r$ is the risk-free interest rate, $\mu$ is the expected return on the log of the risky asset and $\sigma^{2}$ its variance. With CRRA power utility given in (1.2), the equilibrium expected risk-premium can be approximated by (in discrete time)

$$
\mu-r=\gamma \operatorname{cov}\left(\alpha(t)-r, \frac{C(t+1)-C(t)}{C(t)}\right)
$$

In the data, the risk-premium is high, but the covariance of consumption with the risky return is low, hence the only way to reconcile this is to entertain a very high $\gamma$, i.e. a very 
high relative risk aversion for the direct utility function [Cochrane (2006)]. This is the equity-premium puzzle.

We also find it informative to look at other implications of this utility specification. Note that when the direct utility function $U(C)$ is of the CRRA power utility form with constant relative risk aversion $\gamma$, the indirect utility function $V(W)$ is also characterized by constant relative risk aversion of the same degree. The Euler equation can also be expressed in terms of the indirect utility function which would imply

$$
\mu-r=\gamma \operatorname{Cov}\left(\alpha(t)-r, \frac{W(t+1)-W(t)}{W(t)}\right)
$$

As noted by Cochrane (2006), in fact there is no equity premium puzzle for the CRRA indirect utility function. Notice that, in a two asset world where $\phi *$ is the optimal share in the risky asset, (3.3) reduces to:

$$
\mu-r=\gamma \operatorname{Cov}\left(\alpha(t)-r, \frac{\phi^{*} \alpha(t) W(t)+\left(1-\phi^{*}\right) r W(t)}{W(t)}\right)=\gamma \phi^{*} \sigma^{2}
$$

which is just the equation for optimal portfolio allocation

$$
\phi^{*}=\frac{\mu-r}{\gamma \sigma^{2}}
$$

Kocherlakota (1996)'s data exhibit an average risk premium of $6 \%$ with variance of slightly under $3 \%$. Therefore, relative risk aversion of the indirect utility function of anything over 2 is consistent with risky asset shares less than one, with the observed risk premium and volatility of wealth.

The equity risk premium puzzle arises because the volatility of wealth is much greater than the volatility of consumption growth (by more than a factor of ten in Kocherlakota's [1996] data). With CRRA power utility, optimal consumption is 
proportional to wealth. So it would be just as accurate to say that with CRRA

preferences the puzzle is to explain the excessive consumption smoothing.

Meyer and Meyer's (2005) Proposition I is helpful for summarizing this analysis:

$$
R_{V}(W)=R_{U}(C) \frac{d C}{d W} \frac{W}{C}=R_{U}(C) \cdot \varepsilon_{C, W}
$$

where $R_{V \text { or } U}$ is relative risk aversion for either the indirect or direct utility functions and $\varepsilon_{C, W}$ is the elasticity of consumption with respect to wealth. The data tell us that the left hand side of (3.6) needs to be something above and close to 2 . The requirement for consumption smoothing can be stated either as a model where $\varepsilon_{C, W}$ (equal to the marginal propensity to consume out of wealth divided by the average propensity) is substantially below 1, or equivalently and more traditionally, where the relative risk aversion of the direct utility function is much higher than 2 .

But Merton shows that for exponential utility as in (1.1), optimal consumption is also a linear function of wealth, where the marginal propensity to consume out of wealth is the real interest rate and the average propensity varies with the time horizon. For the infinite horizon case, the optimal choice for consumption is given by

$$
C^{*}=\left[\frac{\rho-r+(\mu-r)^{2} / 2 \sigma^{2}}{A r}\right]+r W
$$

Thus the elasticity of consumption with respect to wealth is the real interest times the inverse of the APC. In this case (at least for the point estimates of the data) ${ }^{13}$ the puzzle is not why consumption is so smooth, but rather why is it so volatile. With Kocherlakota's

\footnotetext{
${ }^{13}$ Cochrane (2006) points out that since the equity premium is estimated imprecisely, the confidence interval around the required degree of risk aversion is large.
} 
[1996] estimate of a real T-bill rate of $1 \%$, the variance of consumption growth should be close to zero (this of course ignores the volatility in the "risk-free" interest rate)! Put in the more traditional way and assuming a consumption wealth ratio of somewhere between 3 and 4, (local) relative risk aversion of the indirect utility function of around 2, by (3.6) implies (local) relative risk aversion for the direct utility function of something between 50 and 67.

Notice, if one compares all of the properties of the DRRA power utility model, the complete model has far more trouble with the data. Merton (1992) has shown that for DRRA power utility, the two measures of relative risk aversion can be written as $^{14}$ :

$$
R_{V}(W)=\gamma \frac{W}{W-Z / r} ; R_{U}(C)=\gamma \frac{C}{C-Z}
$$

in Merton's model $Z$ should be thought of as a time invariant subsistence level. At a moment in time, the distinction between subsistence and time-varying habit is unimportant. We will continue to call $C-Z$ surplus consumption, which suggests calling $W-Z / r$ surplus wealth. This measure is just total wealth less the capitalized value of future subsistence needs, using the real interest rate as a discount rate. ${ }^{15}$ Consequently, the ratio of wealth to surplus wealth will be at least as great as the ratio of consumption to surplus consumption. Therefore, the properties of the complete model cannot fit the data. Setting $R_{U}(C)$ high enough to match the consumption, Euler equation implies ridiculously low levels of holdings of the risky asset. This is particularly evident when one recognizes that the amount held in the risky asset is a fraction based on $R_{V}(W)$ times surplus wealth.

\footnotetext{
${ }^{14}$ See Merton(1991), pp 138-9, where our $\gamma$ replaces his $1-\gamma$, our $Z$ replaces his $-\delta \eta$,and his $\beta$ is equal to 1 .

${ }^{15}$ (3.8) uses the infinite horizon version of the model, but the sentence is true for finite horizons as well.
} 
The exponential utility model does not match the point estimates of the data perfectly; in particular the marginal propensity to consume out of wealth seems low, aversion to consumption volatility seems high. However, it is not off by the orders of magnitude of the complete DRRA power utility model. Notice also that the exponential model solves the equity premium puzzle without imposing a high-degree of relative risk aversion on $V(W)$, and it is this indirect utility function that matters when studying issues such as insurance. As opposed to Cochrane's (2006, p28) pessimistic conclusion that the equity premium puzzle can only be solved with high risk aversion, we believe the exponential utility model largely solves the puzzle with low risk aversion.

But the exponential utility model still has a problem: the high implied relative risk aversion for the direct utility function exacerbates the risk-free puzzle. As we show in the next section, the risk-free puzzle can be solved by adopting a form of habit formation where utility depends on consumption relative to wealth.

\section{Exponential Utility with Habit Formation}

As argued in section 3, when preferences are described by the exponential utility function as in (1.1) the optimal choice for consumption and share of risky asset are given by

$$
C^{*}=\left[\frac{\rho-r+(\mu-r)^{2} / 2 \sigma^{2}}{A r}\right]+r W \text { and } \phi^{*}=\frac{(\mu-r)}{A r \sigma^{2} W}
$$

The first implication from the above optimal consumption rule is that these preferences are not consistent with a balanced growth path along which consumption and wealth would grow at the same rate. This is clearly at odds with the data and standard growth theory. On the other hand, as pointed out in section 3, this optimal consumption 
rule is consistent with volatile wealth and smooth consumption observed in the data. One challenge therefore is to generate an optimal consumption rule that would be consistent with a balanced growth, but will still be consistent with volatile wealth and smooth consumption.

The above optimal choice for the share of the risky asset in the portfolio implies that wealth is inversely related to the share of risky asset; this is a direct result of increasing relative risk aversion property of these preferences. The relative risk aversion for the direct and the indirect utility functions are given by

$$
R_{U}(C)=A C \text { and } R_{V}(W)=A r W
$$

which imply that wealthier people are more risk averse which is again at odds with empirical observations.

But the problems with exponential utility disappear if we assume direct utility is a function of consumption relative to some habit level of consumption. Consider the following lifetime portfolio choice problem given by

$$
\begin{aligned}
V\left[\frac{W(t)}{Z(t)}\right]= & \max _{C(t), \phi(t)} E \int_{0}^{\infty} e^{-\rho t} U\left[\frac{C(t)}{Z(t)}\right] d t \\
& \text { s.t. } \frac{d W}{d t}=\{\phi(t)[\alpha(t)-r]+r\} W(t)-C(t) \\
& \text { s.t. } \log \alpha(t) \sim N\left(\mu, \sigma^{2}\right) \\
& \text { s.t. } \frac{d Z}{d t}=\beta \frac{Z(t)}{W(t)}[C(t)-Z(t)]
\end{aligned}
$$


where the last constraint is describing how the habit level of consumption, $Z$, evolves over time. ${ }^{16}$ Redefining the variables as $c=C / Z$ and $w=W / Z$, the portfolio choice problem can be written as:

$$
\begin{aligned}
V[w(t)]= & \max _{C(t), \phi(t)} E \int_{0}^{\infty} e^{-\rho t} U[c(t)] d t \\
& \text { s.t. } \frac{d w}{d t}=\beta+\{\phi(t)[\alpha(t)-r]+r\} w(t)-(1+\beta) c(t)
\end{aligned}
$$

With the direct utility function defined by (1.4) we can show that the indirect utility function is given by

$$
V(w)=-\frac{p}{q} e^{-q w}
$$

where

$$
p=\frac{1}{1+\beta} \exp \left(\frac{r-\rho-\frac{A r \beta}{1+\beta}-\frac{(\mu-r)^{2}}{2 \sigma^{2}}}{r}\right) \text { and } q=\frac{A r}{1+\beta}
$$

The solution to the optimal portfolio problem is given by

$$
c^{*}=\left[\frac{\rho-r+A r \beta /(1+\beta)+(\mu-r)^{2} / 2 \sigma^{2}}{A r}\right]+r w \text { and } \phi^{*}=\frac{(1+\beta)(\mu-r)}{A r \sigma^{2} w}
$$

Note that the optimal consumption rule is now consistent with balanced growth (since the habit level of consumption is growing over time at roughly the same rate), and can still generate volatile wealth with much smoother consumption because the marginal propensity to spend out of wealth is still the real interest rate. Also note that the relative risk aversion factors on the direct and the indirect utility functions are the same as before

\footnotetext{
${ }^{16}$ This evolution for the habit level is not standard in the literature, however we use it in order to derive a closed form solution for the indirect utility function. All derivations of this section is given in the Appendix.
} 
except now they are defined over relative consumption and wealth, hence they can generate roughly constant relative risk aversion across individuals as long as richer people's habit consumptions are proportionally higher.

The last remaining problem is to explain the risk-free rate puzzle. The derivation of the Euler equation for the risk-free rate is tedious and relegated to the Appendix, where it is shown that:

$$
r-\rho=\gamma E\left(g^{W / Z}\right)-\frac{\gamma^{2}}{2} \sigma^{2} \phi^{*}
$$

where $\gamma$ is local relative risk aversion of the indirect utility function and $g^{w / z}$ is growth in relative wealth. Notice that relative risk aversion eliminates the risk-free puzzle by eliminating secular growth in the argument of the utility functions, i.e., $E\left(g^{w / z}\right)$ is roughly zero, and the last term helps to explain the low risk-free interest rate. Again, using Kocherlakota's [1996], (4.7) can be fulfilled with $r=.01, \gamma=2, \phi *=1$, if the pure rate of time preference is $7 \%$.

\section{Conclusion}

In this paper, we laid out a case for reviving the use of exponential utility. CRRA power utility, the workhorse of modern macro macroeconomics and asset-pricing literature, leads to St. Petersburg-style paradoxes due to its unboundedness, is indifferent to compound gambles and generates asset pricing puzzles. Although adding habit formation has solved many of the asset pricing problems of power utility, we think it might be worthwhile to start reconsidering the use of exponential utility.

Exponential utility had been abandoned mainly because it generates increasing relative risk aversion in the cross-section and is not consistent with balanced growth. The 
strengths of exponential are that it is bounded and is consistent with a high risk-premium. We augment the standard exponential utility with habit formation and show that these preferences are bounded, not indifferent to compound gambles, generate near-constant relative risk aversion in the cross-section and are consistent with the high risk-premium and low risk-free rate and are consistent with the volatility of consumption and wealth.

Merton (1969) and Samuelson (1969) clearly made a major contribution by placing portfolio choice in the context of a model based on the utility of consumption. We believe that the profession, by focusing so much attention on the direct utility function, has paid insufficient attention to the model's consistency with the indirect utility function as well as implications for volatility of consumption and wealth. In judging whether a model is broadly consistent with the data, we believe that one must examine all aspects of the model; i.e., direct and indirect utility function and consumption function.

Future research should also concentrate on the business cycle implications of exponential utility with habit formation alongside with the asset pricing implications to ensure applicability of these preferences in a variety of contexts. 


\section{References}

Abel, A. (1990), "Asset Prices Under Habit Formation and Catching Up with the Joneses," American Economic Review, 80, pp. 38-42

Arrow, K., (1971), Essays in the Theory of Risk-Bearing, (Chicago: Markham Publishing Co.)

Campbell, J. and J. Cochrane (1999), "By Force of Hasbit: A Consumption-Based Explanation of Aggregate Stock Market Behavior," Journal of Political Economy, 107, pp. 205-51.

Cochrane, J. (2006), "Financial Markets and the Real Economy," Manuscript, University of Chicago.

Constantinides, G. (1990), "Habit Formation: A Resolution of the Equity Premium Puzzle," The Journal of Political Economy, 98, (June), pp. 519-43.

Davis, M. and R. Martin, (2005) "Housing, House Prices and the Equity Premium Puzzle," Finance and Economics Discussion Series: 2005-13, Board of Governors of the Federal Reserve System

Epstein, L. and S. Zin, (1991), "Substitution, Risk Aversion, and the Temporal Behavior of Asset Returns," Journal of Political Economy, 99, pp. 263-86.

Friend, I. and M. Blume (1975), "The Demand for Risky Assets," American Economic Review, 65 (December), pp. 900-22.

Gali, J. (1994), "Keeping Up with the Joneses: Consumption Externalities, Portfolio Choice, and Asset Prices," Journal of Money Credit and Banking, 26, pp. 1-8.

Kocherlakota, N. (1996), “The Equity Premium: It's Still a Puzzle,” Journal of Economic Literature, 34, (March), pp. 42-71.

Mehra, R. and E. Prescott (1985), “The Equity Premium Puzzle," Journal of Monetary Economics, 15, (March), pp. 145-161.

Merton R., (1969), "Lifetime Portfolio Selection by Dynamic under Uncertainty: The Continuous Time Case,” Review of Economic Statistics, 51 (August), pp. 247-57.

(1992), Continuous Time Finance, (Cambridge: Blackwell Publishing Co.).

Meyer, D. and J. Meyer (2005), "Risk Preferences in Multi-Period Consumption Models, the Equity Premium Puzzle, and Habit Formation Utility," Journal of Monetary Economics, 52, (November), pp. 1497-515.

Samuelson, P. (1963), “A Fallacy of the Law of Large Numbers," Scienta, 6 th series, $57^{\text {th }}$ year, (April-May). 
(1969), "Lifetime Portfolio Selection by Dynamic Stochastic Programming," Review of Economic Studies, 51 (August), pp. 239-46.

Sundaresan, S. (1989), "Intertemporally Dependent Preferences and the Volatility of Consumption and Wealth," Review of Financial Studies, 2, pp. 73-89.

Weil, P. (1989), “The Equity Premium Puzzle and the Risk-Free Rate Puzzle," Journal of Monetary Economics, 24, (November), pp. 401-21. 


\section{Appendix:}

\section{A. Compound Gambles and Relative Risk Aversion:}

Indifference in a one-trial gamble:

$P V(X)+(1-P) V\left(X^{-1}\right)=V(1) \equiv-1$

Two-trial gamble:

$$
\begin{aligned}
& P^{2} V\left(X^{2}\right)+2 P(1-P) V(1)+(1-P)^{2} V\left(X^{-2}\right)= \\
& P^{2} V\left(X^{2}\right)+2 P(1-P) V(X) V\left(X^{-1}\right)+(1-P)^{2} V\left(X^{-2}\right)-2 P(1-P)\left(V(X) V\left(X^{-1}\right)-V(1)\right) \\
& \text { If } \quad-V\left(X^{2}\right)=V(X)^{2} ;-V\left(X^{-2}\right)=V(X)^{-2} ; V(X)^{-1}=V\left(X^{-1}\right) \\
& P^{2} V\left(X^{2}\right)+2 P(1-P) V(X) V\left(X^{-1}\right)+(1-P)^{2} V\left(X^{-2}\right)-2 P(1-P)\left(V(X) V\left(X^{-1}\right)-1\right)= \\
& -\left(P V(X)+(1-P) V\left(X^{-1}\right)\right)^{2}=-1=V(1) \\
& \text { If } V\left(X^{2}\right)>-V(X)^{2} ; V\left(X^{-2}\right)>-V(X)^{-2} ; V\left(X^{-1}\right)>V(X)^{-1} \\
& P^{2} V\left(X^{2}\right)-2 P(1-P) V(X) V\left(X^{-1}\right)+(1-P)^{2} V\left(X^{-2}\right)+2 P(1-P)\left(V(X) V\left(X^{-1}\right)-1\right)> \\
& -P^{2} V(X)^{2}-2 P(1-P) V(X) V\left(X^{-1}\right)-(1-P)^{2} V(X)^{-2}= \\
& -\left(P V(X)+(1-P) V\left(X^{-1}\right)\right)^{2}=-1=V(1) ;
\end{aligned}
$$

Two-trial gamble is preferred.

Necessary conditions so that $-V\left(X^{2}\right)=V(X)^{2} ;-V\left(X^{-2}\right)=V(X)^{-2} ; V(X)^{-1}=V\left(X^{-1}\right)$

$$
\begin{aligned}
& H(X) \equiv \frac{V(X)^{2}}{V\left(X^{2}\right)} ; H(1)=1 ; \\
& \frac{d H}{d X}=\frac{2 V^{\prime}(X) V(X)}{V\left(X^{2}\right)}-\frac{2 X V^{\prime}\left(X^{2}\right) V(X)^{2}}{V\left(X^{2}\right)^{2}} \\
& \frac{d H}{d X} \geq 0 \text { as } \frac{X V^{\prime}(X)}{V(X) V\left(X^{2}\right)}-\frac{X^{2} V^{\prime}\left(X^{2}\right)}{V\left(X^{2}\right)^{2}} \geq 0 \\
& \text { as }-\frac{X V^{\prime}(X)}{V(X)} \geq-\frac{X^{2} V^{\prime}\left(X^{2}\right)}{V\left(X^{2}\right)}
\end{aligned}
$$




$$
\begin{aligned}
& I(X) \equiv \frac{V(X)^{-2}}{V\left(X^{-2}\right)} ; I(1)=1 ; \\
& \frac{d I}{d X}=-\frac{2 V^{\prime}(X) V(X)^{-3}}{V\left(X^{-2}\right)}+\frac{2 X^{-3} V^{\prime}\left(X^{-2}\right) V(X)^{-2}}{V\left(X^{-2}\right)^{2}} \\
& \frac{d I}{d X} \geq 0 \text { as }-\frac{X^{\prime}(X)}{V(X)} \leq-\frac{X^{-2} V^{\prime}\left(X^{-2}\right)}{V\left(X^{-2}\right)} \\
& J(X) \equiv \frac{V(X)^{-1}}{V\left(X^{-1}\right)} ; J(1)=1 ; \\
& \frac{d J}{d X}=-\frac{V^{\prime}(X) V(X)^{-2}}{V\left(X^{-1}\right)}+\frac{X^{-2} V^{\prime}\left(X^{-1}\right) V(X)^{-1}}{V\left(X^{-1}\right)^{2}} \\
& \frac{d J}{d X} \geq 0 \text { as }-\frac{X V^{\prime}(X)}{V(X)} \leq-\frac{X^{-1} V^{\prime}\left(X^{-1}\right)}{V\left(X^{-1}\right)} \\
& \frac{d H}{d X} \geq 0 ; \frac{d I}{d X} \geq 0 ; \frac{d V}{d X} \geq 0 ; \text { if } \frac{X V^{\prime}(X)}{V^{\prime}(X)} / d X
\end{aligned}
$$

A power utility function with increasing relative risk aversion can be written as:

$V(X)=-(X(1-Z)+Z)^{1-\gamma}$,

$(\gamma>1,1>Z>0, V(1)=-1)$,

$-\frac{X V^{\prime}(X)}{V(X)}=-(1-\gamma)(1-Z) \frac{X}{X(1-Z)+Z}$,

which is an increasing function of $X$ as $Z>0$.

An exponetial utility function can be written as: $\mathrm{V}(X)=-e^{-A(X-1)}$, $(A>0, V(1)=-1)$,

$-\frac{X V^{\prime}(X)}{V(X)}=A X$,

which is an increasing function of $X$. 


\section{B. Exponential Utility with Habit Consumption}

The portfolio choice problem is given by:

$$
\begin{aligned}
V\left[\frac{W(t)}{Z(t)}\right]= & \max _{C(t), \phi(t)} E \int_{0}^{\infty} e^{-\rho t} U\left[\frac{C(t)}{Z(t)}\right] d t \\
& \text { s.t. } \frac{d W}{d t}=\{\phi(t)[\alpha(t)-r]+r\} W(t)-C(t) \\
& \text { s.t. } \log \alpha(t) \sim N\left(\mu, \sigma^{2}\right) \\
& \text { s.t. } \frac{d Z}{d t}=\beta \frac{Z(t)}{W(t)}[C(t)-Z(t)]
\end{aligned}
$$

Note that

$$
\begin{aligned}
& \frac{d w}{d t}=\frac{1}{Z(t)} \frac{d W}{d t}-\frac{W(t)}{Z(t)^{2}} \frac{d Z}{d t}=\frac{\{\phi(t)[\alpha(t)-r]+r\} W(t)-C(t)}{Z(t)}-\frac{W(t)}{Z(t)^{2}}\left\{\beta \frac{Z(t)}{W(t)}[C(t)-Z(t)]\right\} \\
& \Rightarrow \frac{d w}{d t}=\{\phi(t)[\alpha(t)-r]+r\} w(t)-c(t)-\beta[c(t)-1] \\
& \Rightarrow \frac{d w}{d t}=\beta+\{\phi(t)[\alpha(t)-r]+r\} w(t)-(1+\beta) c(t)
\end{aligned}
$$

Hence the portfolio choice problem can be written as

$$
\begin{aligned}
V[w(t)]= & \max _{C(t), \phi(t)} E \int_{0}^{\infty} e^{-\rho t} U[c(t)] d t \\
& \text { s.t. } \frac{d w}{d t}=\beta+\{\phi(t)[\alpha(t)-r]+r\} w(t)-(1+\beta) c(t) \\
\text { s.t. } & \log \alpha(t) \sim N\left(\mu, \sigma^{2}\right)
\end{aligned}
$$

Bellman's optimality condition:

$$
0=\max _{c, \phi} U(c)-\rho V(w)+V^{\prime}(w)\{\beta+[\phi(\mu-r)+r] w-(1+\beta) c\}+\frac{1}{2} V^{\prime \prime}(w) \sigma^{2} \phi^{2} w^{2}
$$

Equilibrium conditions: 
(1) $0=U\left(c^{*}\right)-\rho J(w)+V^{\prime}(w)\left\{\beta+\left[\phi^{*}(\mu-r)+r\right] w-(1+\beta) c\right\}+\frac{1}{2} V^{\prime \prime}(w) \sigma^{2} \phi^{*^{2}} w^{2}$

(2) $0=U^{\prime}\left(c^{*}\right)-(1+\beta) V^{\prime}(w)$

(3) $0=(\mu-r) V^{\prime}(w)+V^{\prime \prime}(w) \sigma^{2} \phi^{*} w$

and the transversality condition $\lim _{t \rightarrow \infty} E\left\{e^{-\rho t} V[w(t)]\right\}=0$

\section{Application to exponential utility with habit:}

$U(c)=\frac{e^{-A c}}{-A}=\frac{U^{\prime}(c)}{-A}, \quad U^{\prime}(c)=e^{-A c}, \quad U^{\prime \prime}(c)=-A e^{-A c}$

Equilibrium conditions:

(2) $0=U^{\prime}\left(c^{*}\right)-(1+\beta) V^{\prime}(w) \Rightarrow e^{-A c^{*}}=(1+\beta) V^{\prime}(w) \Rightarrow c^{*}=-\frac{1}{A} \log \left[(1+\beta) V^{\prime}(w)\right]$

(3) $0=(\mu-r) V^{\prime}(w)+V^{\prime \prime}(w) \sigma^{2} \phi^{*} w \Rightarrow \phi^{*}=-\frac{(\mu-r) V^{\prime}(w)}{V^{\prime \prime}(w) \sigma^{2} w}$

(1) $0=U\left(c^{*}\right)-\rho V(w)+V^{\prime}(w)\left\{\beta+\left[\phi^{*}(\mu-r)+r\right] w-(1+\beta) c^{*}\right\}+\frac{1}{2} V^{\prime \prime}(w) \sigma^{2} \phi^{* 2} w^{2}$

$\Rightarrow 0=\frac{U^{\prime}\left(c^{*}\right)}{-A}-\rho V(w)+\beta V^{\prime}(w)+V^{\prime}(w) \phi^{*}(\mu-r) w+V^{\prime}(w) r w-(1+\beta) V^{\prime}(w) c^{*}+\frac{1}{2} V^{\prime \prime}(w) \sigma^{2} \phi^{* 2} w^{2}$

$\Rightarrow 0=-\frac{(1+\beta) V^{\prime}(w)}{A}-\rho V(w)+\beta V^{\prime}(w)+V^{\prime}(w) \phi^{*}(\mu-r) w+V^{\prime}(w) r w-(1+\beta) V^{\prime}(w) c^{*}-\frac{1}{2} V^{\prime}(w) \phi^{*}(\mu-r) w$

$\Rightarrow 0=-\frac{(1+\beta) V^{\prime}(w)}{A}-\rho V(w)+\beta V^{\prime}(w)+V^{\prime}(w) r w+\frac{(1+\beta) V^{\prime}(w)}{A} \log \left[(1+\beta) V^{\prime}(w)\right]+\frac{1}{2} V^{\prime}(w) \phi^{*}(\mu-r) w$

$\Rightarrow 0=-\frac{(1+\beta) V^{\prime}(w)}{A}-\rho V(w)+\beta V^{\prime}(w)+V^{\prime}(W) r w+\frac{(1+\beta) V^{\prime}(w)}{A} \log \left[(1+\beta) V^{\prime}(w)\right]-\frac{(\mu-r)^{2}}{2 \sigma^{2}} \frac{\left[V^{\prime}(w)\right]^{2}}{V^{\prime \prime}(w)}$

\section{Trial solution:}

$V(w)=-\frac{p}{q} e^{-q w}, \quad \mathrm{~V}^{\prime}(w)=p e^{-q w}, \mathrm{~V}^{\prime \prime}(W)=-p q e^{-q w}$ 


$$
\begin{aligned}
& \text { (1) } 0=-\frac{(1+\beta) V^{\prime}(w)}{A}-\rho V(w)+\beta V^{\prime}(w)+V^{\prime}(W) r w+\frac{(1+\beta) V^{\prime}(w)}{A} \log \left[(1+\beta) V^{\prime}(w)\right]-\frac{(\mu-r)^{2}}{2 \sigma^{2}} \frac{\left[V^{\prime}(w)\right]^{2}}{V^{\prime \prime}(w)} \\
& \Rightarrow 0=-\frac{(1+\beta) p}{A} e^{-q w}+\frac{\rho p}{q} e^{-q w}+\beta p e^{-q w}+p r w e^{-q W}+\frac{(1+\beta) p}{A} e^{-q w}[\log (1+\beta)+\log (p)-q w]+\frac{(\mu-r)^{2}}{2 \sigma^{2}} \frac{p}{q} e^{-} \\
& \Rightarrow 0=-\frac{(1+\beta)}{A}+\frac{\rho}{q}+\beta+r w+\frac{(1+\beta)}{A}[\log (1+\beta)+\log (p)-q w]+\frac{(\mu-r)^{2}}{2 \sigma^{2}} \frac{1}{q} \\
& \Rightarrow 0=-\frac{(1+\beta) q}{A}+\rho+\beta q+\left[q r-\frac{(1+\beta) q^{2}}{A}\right] w+\frac{(1+\beta) q}{A} \log (1+\beta)+\frac{(1+\beta) q}{A} \log (p)+\frac{(\mu-r)^{2}}{2 \sigma^{2}}
\end{aligned}
$$

This needs to hold for any $w$, hence it must be the case that

$$
\begin{aligned}
& {\left[q r-\frac{(1+\beta) q^{2}}{A}\right]=0 \Rightarrow q=\frac{A r}{1+\beta}} \\
& \text { (1) } 0=-\frac{(1+\beta) q}{A}+\rho+\beta q+\frac{(1+\beta) q}{A} \log (1+\beta)+\frac{(1+\beta) q}{A} \log (p)+\frac{(\mu-r)^{2}}{2 \sigma^{2}} \\
& \Rightarrow 0=-r+\rho+\frac{A r \beta}{1+\beta}+r \log (1+\beta)+r \log (p)+\frac{(\mu-r)^{2}}{2 \sigma^{2}} \Rightarrow p=\mathrm{e}^{\frac{r-\rho-\frac{A r \beta}{1+\beta}-\log (1+\beta)-\frac{(\mu-r)^{2}}{2 \sigma^{2}}}{r}} \\
& \Rightarrow p=\frac{1}{1+\beta} \mathrm{e}^{\frac{r-\rho-\frac{A r \beta}{1+\beta}-\frac{(\mu-r)^{2}}{2 \sigma^{2}}}{r}}
\end{aligned}
$$

Solution:

$$
\begin{aligned}
& c^{*}=-\frac{1}{A} \log \left[(1+\beta) V^{\prime}(w)\right]=-\frac{1}{A}\{\log [(1+\beta) p]-q w\}=-\frac{1}{A r}\left[r-\rho-\frac{A r \beta}{1+\beta}-\frac{(\mu-r)^{2}}{2 \sigma^{2}}\right]+r w \\
& \Rightarrow c^{*}=\left[\frac{\rho-r+A r \beta /(1+\beta)+(\mu-r)^{2} / 2 \sigma^{2}}{A r}\right]+r w \\
& \phi^{*}=-\frac{(\mu-r) V^{\prime}(w)}{V^{\prime \prime}(w) \sigma^{2} w}=\frac{(\mu-r)}{q \sigma^{2} w}=\frac{(1+\beta)(\mu-r)}{A r \sigma^{2} w}
\end{aligned}
$$




\section{The Euler Equation for the Risk-free Rate in Continuous Time}

In discrete time our model can be written as:

$$
\begin{aligned}
& V\left(\frac{W_{t}}{Z_{t}}\right)=U\left(\frac{C^{*}}{Z_{t}}\right) h+e^{-\rho h} E\left[V\left(\frac{W_{t+h}}{Z_{t+h}}\right)\right] ; \\
& \frac{W_{t+h}}{Z_{t+h}}-\frac{W_{t}}{Z_{t}}=\frac{W_{t+h}}{Z_{t+h}}-\frac{W_{t}}{Z_{t+h}}+\frac{W_{t}}{Z_{t+h}}-\frac{W_{t}}{Z_{t}}=\frac{\Delta W_{t+h}}{Z_{t+h}}-\frac{W_{t} \Delta Z_{t+h}}{Z_{t} Z_{t+h}} \\
& \Delta Z_{t+h}=\beta \frac{Z_{t}}{W_{t}}\left[C_{t}-Z_{t}\right] h \\
& \frac{W_{t+h}}{Z_{t+h}}-\frac{W_{t}}{Z_{t}}=\frac{\Delta W_{t+h}}{Z_{t+h}}-\beta \frac{\left[C_{t}-Z_{t}\right]}{Z_{t+h}} h \\
& U^{\prime}\left(\frac{C_{t}^{*}}{Z_{t}}\right) \frac{h}{Z_{t}}-e^{-\rho h} E\left[V^{\prime}\left(\frac{W_{t+h}}{Z_{t+h}}\right)\right]\left[\frac{e^{r h}+\beta h}{Z_{t+h}}\right]=0
\end{aligned}
$$

limit as $h \rightarrow 0$

$$
\begin{aligned}
& U^{\prime}\left(\frac{C_{t}^{*}}{Z_{t}}\right)-(1+r-\rho+\beta) V^{\prime}\left(\frac{W_{t}}{Z_{t}}\right)-V^{\prime \prime}\left(\frac{W_{t}}{Z_{t}}\right) \frac{W_{t}}{Z_{t}} E\left(\frac{\Delta \frac{W_{t}}{Z_{t}}}{\left.\frac{W_{t}}{Z_{t}}\right)}\right) E\left[V^{\prime \prime \prime}\left(\frac{W_{t}}{Z_{t}}\right)\left(\frac{W_{t}}{Z_{t}}\right)^{2}\left(\frac{\Delta \frac{W_{t}}{Z_{t}}}{\frac{W_{t}}{Z_{t}}}\right)^{2}\right] \\
& U^{\prime}\left(\frac{C_{t}^{*}}{Z_{t}}\right)-(1+r-\rho+\beta) V^{\prime}\left(\frac{W_{t}}{Z_{t}}\right)-V^{\prime \prime}\left(\frac{W_{t}}{Z_{t}}\right) \frac{W_{t}}{Z_{t}} E\left(g_{t}^{\frac{W}{Z}}\right)+V^{\prime \prime \prime}\left(\frac{W_{t}}{Z_{t}}\right)\left(\frac{W_{t}}{Z_{t}}\right)^{2} \frac{\sigma^{2} \phi^{*}}{2}
\end{aligned}
$$

In continuous time $U^{\prime}\left(\frac{C_{t}^{*}}{Z_{t}}\right)=(1+\beta) V^{\prime}\left(\frac{W_{t}}{Z_{t}}\right)$

$1=\frac{(1+r-\rho+\beta)}{1+\beta}-\frac{\gamma E\left(g_{t}^{\frac{W}{Z}}\right)}{1+\beta}+\frac{\gamma^{2}}{2} \frac{\sigma^{2} \phi^{*}}{1+\beta}$

where $\gamma$ is local relative risk aversion.

$$
\begin{aligned}
& 1+\beta=(1+r-\rho+\beta)-\gamma E\left(g_{t}^{\frac{W}{Z}}\right)+\gamma^{2} \sigma^{2} \phi^{*} \\
& r-\rho=\gamma E\left(g_{t}^{\frac{W}{Z}}\right)-\frac{\gamma^{2}}{2} \sigma^{2} \phi^{*}
\end{aligned}
$$

The last line above is (4.7) in the text. 